\title{
Foot Complications in a Representative Australian Inpatient Population
}

\author{
Peter A. Lazzarini, ${ }^{1,2,3,4}$ Sheree E. Hurn, ${ }^{1,2}$ Suzanne S. Kuys, ${ }^{3,5}$ Maarten C. Kamp, ${ }^{1}$ \\ Vanessa Ng, ${ }^{3}$ Courtney Thomas, ${ }^{6}$ Scott Jen, ${ }^{7}$ Jude Wills, ${ }^{8}$ Ewan M. Kinnear, ${ }^{3}$ \\ Michael C. d'Emden, ${ }^{1,9}$ and Lloyd F. Reed ${ }^{1,2}$ \\ ${ }^{1}$ School of Clinical Sciences, Queensland University of Technology, Brisbane, QLD, Australia \\ ${ }^{2}$ Institute of Health and Biomedical Innovation, Queensland University of Technology, Brisbane, QLD, Australia \\ ${ }^{3}$ Allied Health Research Collaborative, Metro North Hospital \& Health Service, Brisbane, QLD, Australia \\ ${ }^{4}$ Wound Management Innovation Cooperative Research Centre, Brisbane, QLD, Australia \\ ${ }^{5}$ Faculty of Health Sciences, School of Physiotherapy, Australian Catholic University, Brisbane, QLD, Australia \\ ${ }^{6}$ Department of Podiatry, North West Hospital \& Health Service, Mount Isa, QLD, Australia \\ ${ }^{7}$ Department of Podiatry, West Moreton Hospital \& Health Service, Queensland Health, Ipswich, QLD, Australia \\ ${ }^{8}$ Department of Podiatry, Central Queensland Hospital \& Health Service, Rockhampton, QLD, Australia \\ ${ }^{9}$ Department of Endocrinology \& Diabetes, Metro North Hospital \& Health Service, Brisbane, QLD, Australia
}

Correspondence should be addressed to Peter A. Lazzarini; peter.lazzarini@health.qld.gov.au

Received 18 May 2017; Accepted 18 September 2017; Published 15 October 2017

Academic Editor: Patrizio Tatti

Copyright (c) 2017 Peter A. Lazzarini et al. This is an open access article distributed under the Creative Commons Attribution License, which permits unrestricted use, distribution, and reproduction in any medium, provided the original work is properly cited.

\begin{abstract}
We investigated the prevalence and factors independently associated with foot complications in a representative inpatient population (adults admitted for any reason with and without diabetes). We analysed data from the Foot disease in inpatients study, a sample of 733 representative inpatients. Previous amputation, previous foot ulceration, peripheral arterial disease (PAD), peripheral neuropathy $(\mathrm{PN})$, and foot deformity were the foot complications assessed. Sociodemographic, medical, and foot treatment history were collected. Overall, $46.0 \%$ had a foot complication with $23.9 \%$ having multiple; those with diabetes had higher prevalence of foot complications than those without diabetes $(p<0.01)$. Previous amputation (4.1\%) was independently associated with previous foot ulceration, foot deformity, cerebrovascular accident, and past surgeon treatment $(p<0.01)$. Previous foot ulceration $(9.8 \%)$ was associated with PN, PAD, past podiatry, and past nurse treatment $(p<0.02)$. PAD $(21.0 \%)$ was associated with older age, males, indigenous people, cancer, PN, and past surgeon treatment $(p<0.02)$. PN $(22.0 \%)$ was associated with older age, diabetes, mobility impairment, and PAD $(p<0.05)$. Foot deformity (22.4\%) was associated with older age, mobility impairment, past podiatry treatment, and PN $(p<0.01)$. Nearly half of all inpatients had a foot complication. Those with foot complications were older, male, indigenous, had diabetes, cerebrovascular accident, mobility impairment, and other foot complications or past foot treatment.
\end{abstract}

\section{Introduction}

Active foot disease (ulcers, infection, or ischaemia) is commonly precipitated by the foot complications of previous amputations, previous foot ulcers, peripheral arterial disease (PAD), peripheral neuropathy (PN), and foot deformity in both diabetes and nondiabetes populations [1-4]. These foot complications not only increase the risk of developing active foot disease in the community but also have been found to increase the risk of developing active foot disease, falls, and pressure injuries in the inpatient setting $[3,5-8]$. Thus, it seems important for clinicians, researchers, and policy makers to understand how often these foot complications present and what other factors may precipitate them in inpatient populations.

Studies investigating foot complications in inpatient populations have predominantly focused within diabetes and 
geriatric inpatient populations [4-9]. These studies suggest that up to $80 \%$ of diabetes inpatients, and up to $50 \%$ of geriatric inpatients, have at least one of these foot complications [4-9]. A recent systematic review of this field found previous studies have investigated either a single foot complication (such as PAD) in a representative adult inpatient population (defined as a typical hospital's inpatient population inclusive of patients admitted for any reason, with or without diabetes and of any age) or multiple foot complications in a specific inpatient population (such as all foot complications in diabetes inpatients only) [3]. This review concluded that no previous study had investigated the prevalence or factors associated with multiple foot complications in a representative inpatient population [3]. Thus, the primary aim of this study was to investigate the point prevalence and factors independently associated with foot complications in a representative adult inpatient population. A secondary aim was to investigate if there were any differences in the prevalence of foot complications between diabetes and nondiabetes inpatients.

\section{Materials and Methods}

This study was a secondary analysis of data collected from the Foot disease in inpatients study, a large multisite observational point prevalence study with the aim of investigating active foot disease and other foot-related conditions in a representative inpatient population $[1,2]$. Previous papers from the Foot disease in inpatients study have investigated and reported on the outcomes of (i) primary and secondary admissions for any foot-related conditions [1] and (ii) the presence of active foot disease (ulcers, infection, and ischaemia) [2]. Thus, the design and methodology of the Foot disease in inpatients study that form the basis of this paper have been described in detail elsewhere $[1,2]$. This paper now aims to investigate and report on the outcomes of the presence of different foot complications (previous amputations, previous foot ulcers, $\mathrm{PAD}, \mathrm{PN}$, and foot deformity).

In brief, the Foot disease in inpatients study investigated all adult inpatients present (in hospital at the time of the study for any medical reason) in five representative public hospitals in Queensland (considered representative of the five different categories of Australian hospitals) on one designated day and they were all invited to participate on that same day, excluding those with a cognitive deficit and those in a maternity or psychiatric ward [1]. Of a total of 1146 inpatients present on those days, 883 were eligible for the study and 733 consented to participate [1]. Those eligible participants who consented reported no age or sex differences to those who did not consent [1]. This sample of 733 inpatients has been reported to be highly reflective of the demographic, social determinant, medical history, and reason for admission characteristics reported elsewhere for representative inpatient populations present in Australia and in other developed nations $[1,2,10]$. Highly trained and tested data collectors collected all data on the 733 participants by surveying each participant to determine their selfreported medical history and physically assessing their feet to clinically diagnose any foot-related conditions $[1,11]$. All data were captured on a validated data collection instrument (the Queensland Foot Disease Form) $[1,2]$.

The explanatory variables for this study were grouped into the domains of participant demographics (age and sex), social determinants (socioeconomic status, geographical remoteness, education levels, country of birth, and indigenous status), medical condition history (diabetes, hypertension, dyslipidaemia, myocardial infarct, cerebrovascular accident, chronic kidney disease, smoking, cancer, arthritis, depression, and acute foot trauma), self-care ability (mobility impairment, vision impairment, and main footwear worn inside and outside the home), and past foot treatment in the year prior to hospitalisation (by podiatrist, general practitioner, specialist physician, surgeon, nurse, orthotist, and other) [1,2]. All variables have been defined in detail elsewhere $[1,2,11]$.

The foot complication outcome variables for this study were previous amputation, previous foot ulceration, peripheral arterial disease (PAD), peripheral neuropathy $(\mathrm{PN})$, and foot deformity [1, 2, 11-17]. All foot complication outcome variables were defined, assessed, and reported according to national and international standards for reporting research on the prevention and management of foot ulcers [13-16] and have been defined in detail elsewhere $[1,2,11]$. Previous amputation was diagnosed as a healed amputation site on the lower extremity (foot or leg) during the clinical examination [1, 2, 12-14]. Previous foot ulceration was diagnosed as a self-reported foot ulcer that had healed, and this was verified during the clinical examination $[1,2,12-14]$. PAD was diagnosed as the absence of at least one-foot pulse with a toe systolic pressure of $<70 \mathrm{mmHg}[1,2,11,14-16]$. Peripheral neuropathy was diagnosed as the failure to perceive the sensation of a 10-gram monofilament on at least two of three plantar forefoot sites on one foot $[1,2,11-14,16]$. Foot deformity was diagnosed as having at least three of the following deformity characteristics on one foot: small muscle wastage, bony prominence, prominent metatarsal heads, hammer or claw toes, limited joint mobility, or Charcot deformity $[1,2,11,13,17]$.

2.1. Statistical Analysis. All data were analysed using SPSS 22.0 for Windows (SPSS Inc., Chicago, IL, USA) or GraphPad Software. Descriptive statistics were used to display all variables. Prevalence with 95\% confidence intervals (95\% CI) was evaluated for all foot complication outcome variables. Pearson's chi-square tests and Student's t-tests were used to test for differences in categorical variables (proportions) and continuous variables (mean (standard deviation)), respectively. Mann-Whitney $U$ tests were also used to test for differences in continuous variables (median (interquartile ranges)) if they were identified not to be normally distributed using Kolmogorov-Smirnov tests. Univariate logistic regression was used to test for crude associations between all explanatory variables and each foot complication outcome $(p<0.05)$. All explanatory variables achieving a statistical significance of $p<0.2$, except those deemed illogical to be potentially on a causal pathway, were included in backwards stepwise multivariate logistic regression analysis 
TABle 1: Proportion of the diabetes and nondiabetes participants with foot complications.

\begin{tabular}{|c|c|c|c|c|}
\hline Foot complication & $\begin{array}{c}\text { All } \\
n(\%(95 \% \text { CI }))\end{array}$ & $\begin{array}{c}\text { Diabetes } \\
n(\%(95 \% \mathrm{CI}))\end{array}$ & $\begin{array}{c}\text { Nondiabetes } \\
n(\%(95 \% \text { CI }))\end{array}$ & $p$ value \\
\hline Participants & 733 & 172 & 561 & \\
\hline Foot complication $(\mathrm{s})^{\mathrm{a}}$ & $336(46.0 \%(42.4-49.7))$ & $112(65.5 \%(58.1-72.2)$ & $224(40.1 \%(36.2-44.3))$ & $<0.001^{*}$ \\
\hline Multiple foot complications ${ }^{\mathrm{b}}$ & $175(23.9 \%(20.9-27.1))$ & $66(38.6 \%(31.6-46.1))$ & $109(19.5 \%(16.4-23.0))$ & $<0.001^{*}$ \\
\hline Previous foot disease ${ }^{c}$ & $81(11.1 \%(9.0-13.6))$ & $38(22.1 \%(16.5-28.9))$ & $43(7.7 \%(5.7-10.2))$ & $<0.001^{*}$ \\
\hline Previous amputation & $30(4.1 \%(2.9-5.8))$ & $16(9.3 \%(5.7-14.7))$ & $14(2.5 \%(1.4-4.2)))$ & $<0.001^{*}$ \\
\hline Previous foot ulcer & $72(9.8 \%(7.9-12.2)$ & $35(20.3 \%(15.0-27.0))$ & $37(6.6 \%(4.8-9.0)))$ & $<0.001^{*}$ \\
\hline Peripheral arterial disease & $153(21.0 \%(18.2-24.1))$ & $60(35.1 \%(28.3-42.5))$ & $93(16.7 \%(13.8-20.0))$ & $<0.001^{*}$ \\
\hline Peripheral neuropathy & $160(22.0 \%(19.1-25.1))$ & $74(43.3 \%(36.1-50.8))$ & $86(15.4 \%(12.6-18.6))$ & $<0.001^{*}$ \\
\hline Foot deformity & $158(22.4 \%(19.5-25.6))$ & $51(30.5 \%(24.0-37.9))$ & $107(19.9 \%(16.7-23.5))$ & $0.004^{*}$ \\
\hline
\end{tabular}

${ }^{*} p<0.05$. ${ }^{a}$ Participants with at least one foot complication. ${ }^{b}$ Participants with two or more foot complications. ${ }^{c}$ Participants with previous foot disease (either previous foot ulcer or previous amputation); CI: confidence interval.

for that foot complication outcome until only variables reaching statistical significance remained $(p<0.05)$ (unadjusted model) $[1,18,19]$. All omitted variables from the unadjusted models were reentered and retained in the models as confounders if the beta effect estimates of any unadjusted independent explanatory variable changed by $>20 \%$ (adjusted model) $[1,18,19]$. Missing data were treated by excluding cases with any missing data in all models as the proportion of missing data cases was minimal ( $<5 \%$ in most cases) $[1,18,19]$.

\section{Results}

Table 1 displays the prevalence proportions (95\% CI) for all foot complications in all participants $(n=733)$, including diabetes participants $(n=172)$ and nondiabetes participants $(n=561)$. Overall, 336 participants $(46.0 \%(95 \%$ CI: 42.4-49.7\%)) had at least one foot complication, including 175 (23.9\% (20.9-27.1\%)) with multiple foot complications and $81(11.1 \%(9.0-13.6 \%))$ with a history of previous foot disease (previous amputation or foot ulceration). Diabetes participants had fewer numbers with a foot complication $(n=112)$ than nondiabetes participants $(n=224)$. Yet diabetes participants had much higher proportions of foot complications (65.5\%) than nondiabetes participants $(40.1 \%)$, including those with multiple foot complications $(38.6 \%$ diabetes, $19.5 \%$ nondiabetes) and previous foot disease $(22.1 \%$ diabetes, $7.7 \%$ nondiabetes $)$ (all $p<0.001$ ).

3.1. Previous Amputation. Thirty participants (4.1\% (2.9$5.8 \%)$ ) had a previous amputation (Table 1), including 16 $(2.2 \%(1.3-3.6 \%))$ with diabetes and $24(3.3 \%(2.2-4.9 \%))$ over 60 years old of total participants (Table 2). After univariate analysis, 19 explanatory variables were associated with previous amputations (all, $p<0.05$ ) (Table 2). Adjusting for the identified confounder of geographical remoteness, a previous amputation was independently associated with previous foot ulcer (odds ratio (95\% CI)) (22.0 (6.9-70.4)), past foot treatment by a surgeon $(10.7(2.9-40.1))$, cerebrovascular accident (CVA) history (6.8 (1.9-25.2)), and foot deformity (5.6 (1.9-16.5)) (all, $p<0.01)$ (Table 3).
3.2. Previous Foot Ulcer. Previous foot ulcers were present in 72 participants $(9.8 \%(7.9-12.2 \%))$ (Table 1$)$, including 35 (4.8\% (3.4-6.6\%)) with diabetes and 48 (6.6\% (5.0-8.6\%)) over 60 years old (Table 2). After univariate analysis, 14 explanatory variables were associated with previous foot ulcers (all, $p<0.05$ ) (Table 2). Adjusting for the identified confounders of geographical remoteness and socioeconomic status, a previous foot ulcer was independently associated with past foot treatment by a nurse (18.8 (5.1-68.7)), PAD (3.9 (2.1-7.1)), PN (3.7 (2.1-6.8)), and past foot treatment by a podiatrist $(2.9(1.6-5.2))($ all, $p<0.01)$ (Table 4$)$.

3.3. Peripheral Arterial Disease. Peripheral arterial disease (PAD) was present in 153 participants $(21.0 \%$ (18.2$24.1 \%)$ ) (Table 1), including $60(8.2 \%(6.4-10.5 \%))$ with diabetes and $121(16.6 \%(14.1-19.5 \%))$ over 60 years old (Table 5). After univariate analysis, 22 explanatory variables were associated with PAD (all, $p<0.05$ ) (Table 5). Adjusting for the identified confounders of past podiatry treatment, PAD was independently associated with older age groups (41-60 years $(4.7(1.5-14.2)), 61-80$ years (8.9 (3.0-26.3)), and $81+$ years $(12.7(4.1-39.4)))$, past foot treatment by a surgeon (5.2 (2.3-11.5)), indigenous status (3.1 (1.4-7.2)), PN (2.3 (1.5-3.4)), male gender (1.7 (1.1-2.6)), and cancer history $(0.5(0.3-0.8))$ (all, $p<0.01)$ (Table 6).

3.4. Peripheral Neuropathy. Peripheral neuropathy (PN) was present in 160 participants $(22.0 \%$ (19.1-25.1\%)) (Table 1), including $74(10.2 \%(8.2-12.6 \%))$ with diabetes and 124 $(17.0 \%(14.5-19.9 \%))$ over 60 years old (Table 5). After univariate analysis, 16 explanatory variables were associated with PN (all, $p<0.05$ ) (Table 5). Adjusting for the identified confounder of geographical remoteness, PN was independently associated with older age groups (41-60 years (2.8 (1.0-7.6)), 61-80 years $(4.5(1.7-11.8))$, and $81+$ years (4.4 (1.6-12.3))), diabetes (3.9 (2.5-6.1)), mobility impairment (3.4 (2.2-5.2)), and PAD (2.1 (1.3-3.2)) (all, $p<0.05)$ (Table 7).

3.5. Foot Deformity. Foot deformity was present in 158 participants $(22.4 \%(19.5-25.6 \%))$ (Table 1), including 
TABLe 2: Participant characteristics and univariate analysis for previous amputation and previous foot ulcer.

\begin{tabular}{|c|c|c|c|c|c|c|c|}
\hline \multirow{2}{*}{ Variables } & \multirow{2}{*}{ All } & \multicolumn{3}{|c|}{ Previous amputation } & \multicolumn{3}{|c|}{ Previous foot ulcer } \\
\hline & & $n(\%)$ & Odds ratio $(95 \% \mathrm{CI})$ & $p$ value & $n(\%)$ & Odds ratio $(95 \% \mathrm{CI})$ & $p$ value \\
\hline Participants & 733 & $30 / 731(4.1 \%)$ & & & $72 / 731(9.8 \%)$ & & \\
\hline \multicolumn{8}{|l|}{ Demographics } \\
\hline Age: mean (SD) years & $62.0(18.6)$ & $71.4(11.1)$ & $1.04(1.01-1.06)$ & $0.006^{* *}$ & $65.8(15.6)$ & $1.01(1.00-1.03)$ & $0.074^{*}$ \\
\hline Age: median (IQR) years & $65(50-76)$ & $72(66-79)$ & & $0.006^{* *}$ & $69(57-76)$ & & $0.128^{*}$ \\
\hline Age groups & & & & NA & & & 0.110 \\
\hline $18-40$ years & $110(15.0 \%)$ & 0 & 1.00 & & $5(6.9 \%)$ & 1.00 & \\
\hline $41-60$ years & $188(25.7 \%)$ & $6(20.0 \%)$ & & & $19(26.4 \%)$ & $2.38(0.86-6.55)$ & 0.095 \\
\hline $61-80$ years & $316(43.2 \%)$ & $17(56.7 \%)$ & & & $39(54.2 \%)$ & $2.97(1.14-7.73)$ & 0.026 \\
\hline $81+$ years & $117(16.0 \%)$ & $7(23.3 \%)$ & & & $9(12.5 \%)$ & $1.75(0.57-5.39)$ & 0.330 \\
\hline Male sex & $408(55.8)$ & $19(63.3 \%)$ & $1.38(0.65-2.95)$ & 0.400 & $46(63.9 \%)$ & $1.46(0.88-2.42)$ & $0.142^{*}$ \\
\hline \multicolumn{8}{|l|}{ Social determinants } \\
\hline Socioeconomic status & 711 & & & 0.638 & & & $0.064^{*}$ \\
\hline Most disadvantaged & $102(14.4 \%)$ & $6(20.7 \%)$ & 1.00 & & $16(22.9 \%)$ & 1.00 & \\
\hline $\begin{array}{l}\text { Second most } \\
\text { disadvantaged }\end{array}$ & $159(22.4 \%)$ & $7(24.1 \%)$ & $0.74(0.24-2.26)$ & 0.593 & $17(24.3 \%)$ & $0.64(0.31-1.34)$ & 0.239 \\
\hline Middle & $98(13.8 \%)$ & $2(6.9 \%)$ & $0.33(0.07-1.69)$ & 0.185 & $4(5.7 \%)$ & $0.23(0.07-0.72)$ & 0.011 \\
\hline $\begin{array}{l}\text { Second least } \\
\text { disadvantaged }\end{array}$ & $240(33.8 \%)$ & $11(37.9 \%)$ & $0.78(0.28-2.16)$ & 0.626 & $26(37.1 \%)$ & $0.66(0.34-1.28)$ & 0.218 \\
\hline Least disadvantaged & $112(15.8 \%)$ & $3(10.3 \%)$ & $0.44(0.11-1.81)$ & 0.255 & $7(10.0 \%)$ & $0.36(0.14-0.91)$ & 0.031 \\
\hline Geographic remoteness & 711 & & & 0.589 & & & 0.304 \\
\hline Major city & $435(61.2 \%)$ & $18(62.1 \%)$ & 1.00 & & $41(58.6 \%)$ & 1.00 & \\
\hline Inner regional area & $153(21.5 \%)$ & $4(13.8 \%)$ & $0.62(0.21-1.86)$ & 0.392 & $15(21.4 \%)$ & $1.04(0.56-1.94)$ & 0.904 \\
\hline Outer regional area & $66(9.3 \%)$ & $5(17.2 \%)$ & $1.89(0.68-5.28)$ & 0.224 & $12(17.1 \%)$ & $2.13(1.05-4.29)$ & 0.036 \\
\hline Remote area & $30(4.2 \%)$ & $1(3.4 \%)$ & $0.80(0.10-6.17)$ & 0.826 & 0 & 0 & NA \\
\hline Very remote area & $27(3.8 \%)$ & $1(3.4 \%)$ & $0.89(0.11-6.90)$ & 0.909 & $2(2.9 \%)$ & $0.77(0.18-3.35)$ & 0.722 \\
\hline$<10$-year education level & $395(54.0 \%)$ & $19(63.3 \%)$ & $1.48(0.70-3.16)$ & 0.307 & $41(56.9 \%)$ & $1.13(0.69-1.85)$ & 0.621 \\
\hline Indigenous & $34(4.6 \%)$ & $1(3.3 \%)$ & $0.70(0.09-5.27)$ & 0.727 & $4(5.6 \%)$ & $1.23(0.42-3.60)$ & 0.704 \\
\hline Born overseas & $161(22.0 \%)$ & $5(16.7 \%)$ & $0.70(0.26-1.85)$ & 0.467 & $11(15.3 \%)$ & $0.61(0.31-1.19)$ & $0.146^{*}$ \\
\hline \multicolumn{8}{|l|}{ Medical condition history } \\
\hline Diabetes & $172(23.5 \%)$ & $16(53.3 \%)$ & $3.99(1.91-8.36)$ & $<0.001^{* *}$ & $35(48.6 \%)$ & $3.60(2.19-5.94)$ & $<0.001^{* *}$ \\
\hline Hypertension & $359(49.0 \%)$ & $21(70.0 \%)$ & $2.54(1.15-5.61)$ & $0.022^{* *}$ & $38(52.8 \%)$ & $1.18(0.73-1.93)$ & 0.497 \\
\hline Dyslipidaemia & $234(31.9 \%)$ & $12(40.0 \%)$ & $1.44(0.68-3.04)$ & 0.341 & $27(37.5 \%)$ & $1.31(0.79-2.17)$ & 0.294 \\
\hline Myocardial infarct & $146(19.9 \%)$ & $12(40.0 \%)$ & $2.82(1.33-6.00)$ & $0.007^{* *}$ & $17(23.6 \%)$ & $1.27(0.71-2.26)$ & 0.417 \\
\hline Cerebrovascular accident & $85(11.6 \%)$ & $9(30.0 \%)$ & $3.52(1.59-7.97)$ & $0.002^{* *}$ & $8(11.1 \%)$ & $0.95(0.44-2.05)$ & 0.885 \\
\hline Chronic kidney disease & $89(12.1 \%)$ & $11(36.7 \%)$ & $4.62(2.12-10.08)$ & $<0.001^{* *}$ & $19(26.4 \%)$ & $3.02(1.69-5.39)$ & $<0.001^{* *}$ \\
\hline Smoker & $104(14.2 \%)$ & $3(10.0 \%)$ & $0.66(0.20-2.22)$ & 0.501 & $12(16.7 \%)$ & $1.23(0.64-2.38)$ & 0.533 \\
\hline Ex-smoker & $304(41.5 \%)$ & $14(46.7 \%)$ & $1.25(0.60-2.60)$ & 0.554 & $28(38.9 \%)$ & $0.89(0.54-1.46)$ & 0.642 \\
\hline Cancer & $174(23.7 \%)$ & $8(26.7 \%)$ & $1.17(0.51-2.68)$ & 0.707 & $17(23.6 \%)$ & $0.99(0.56-1.75)$ & 0.968 \\
\hline Arthritis & $274(37.4 \%)$ & $18(60.0 \%)$ & $2.64(1.25-5.57)$ & $0.011^{* *}$ & $41(56.9 \%)$ & $2.43(1.49-3.99)$ & $<0.001^{* *}$ \\
\hline Depression & $191(26.1 \%)$ & $6(20.0 \%)$ & $0.70(0.28-1.75)$ & 0.447 & $21(29.2 \%)$ & $1.18(0.69-2.03)$ & 0.537 \\
\hline Acute foot trauma & $26(3.5 \%)$ & $1(3.3 \%)$ & $0.93(0.12-7.12)$ & 0.946 & $7(9.7 \%)$ & $3.63(1.47-8.95)$ & $0.005^{* *}$ \\
\hline \multicolumn{8}{|l|}{ Self-care ability } \\
\hline Mobility impairment & $242(33.2 \%)$ & $21(70.0 \%)$ & $5.07(2.29-11.25)$ & $<0.001^{* *}$ & $38(52.8 \%)$ & $2.48(1.52-4.05)$ & $<0.001^{* *}$ \\
\hline Vision impairment & $110(15.1 \%)$ & $12(40.0 \%)$ & $4.09(1.91-8.75)$ & $<0.001^{* *}$ & $20(27.8 \%)$ & $2.42(1.38-4.25)$ & $0.002^{* *}$ \\
\hline Footwear worn: inside & & & & $0.158^{*}$ & & & 0.580 \\
\hline Low-risk footwear & $81(11.1 \%)$ & $6(20.7 \%)$ & 1.00 & & $11(15.5 \%)$ & 1.00 & \\
\hline
\end{tabular}


TABLE 2: Continued.

\begin{tabular}{|c|c|c|c|c|c|c|c|}
\hline \multirow{2}{*}{ Variables } & \multirow{2}{*}{ All } & \multicolumn{3}{|c|}{ Previous amputation } & \multicolumn{3}{|c|}{ Previous foot ulcer } \\
\hline & & $n(\%)$ & Odds ratio $(95 \% \mathrm{CI})$ & $p$ value & $n(\%)$ & Odds ratio $(95 \% \mathrm{CI})$ & $p$ value \\
\hline $\begin{array}{l}\text { Moderate-risk } \\
\text { footwear }\end{array}$ & $263(36.1 \%)$ & $13(44.8 \%)$ & $0.65(0.24-1.77)$ & 0.399 & $27(38.0 \%)$ & $0.73(0.34-1.54)$ & 0.407 \\
\hline High-risk footwear & $139(19.1 \%)$ & $2(6.9 \%)$ & $0.18(0.04-0.93)$ & 0.041 & $12(16.9 \%)$ & $0.61(0.25-1.45)$ & 0.259 \\
\hline No footwear worn & $245(33.7 \%)$ & $8(27.6 \%)$ & $0.42(0.14-1.26)$ & 0.121 & $21(29.6 \%)$ & $0.60(0.27-1.30)$ & 0.193 \\
\hline Footwear worn: outside & & & & $0.116^{*}$ & & & 0.235 \\
\hline Low-risk footwear & $386(53.2 \%)$ & $21(75.0 \%)$ & 1.00 & & $36(50.7 \%)$ & 1.00 & \\
\hline $\begin{array}{l}\text { Moderate-risk } \\
\text { footwear }\end{array}$ & $75(10.3 \%)$ & $1(3.6 \%)$ & $0.23(0.03-1.77)$ & 0.159 & $11(15.5 \%)$ & $1.67(0.81-3.44)$ & 0.168 \\
\hline High-risk footwear & $250(34.4 \%)$ & $5(17.9 \%)$ & $0.35(0.13-0.95)$ & 0.039 & $21(29.6 \%)$ & $0.89(0.51-1.56)$ & 0.682 \\
\hline No footwear worn & $15(2.1 \%)$ & $1(3.6 \%)$ & $1.24(0.16-9.87)$ & 0.840 & $3(4.2 \%)$ & $2.42(0.65-8.99)$ & 0.186 \\
\hline \multicolumn{8}{|l|}{ Past foot treatment } \\
\hline Yes & $256(34.9 \%)$ & $22(73.3 \%)$ & $5.52(2.42-12.60)$ & $<0.001^{* *}$ & $56(77.8 \%)$ & $8.03(4.50-14.35)$ & $<0.001^{* *}$ \\
\hline Podiatry & $180(24.6 \%)$ & $18(60.0 \%)$ & $50.3(2.37-10.67)$ & $<0.001^{* *}$ & $41(56.9 \%)$ & $4.95(2.99-8.18)$ & $<0.001^{* *}$ \\
\hline GP & $93(12.7 \%)$ & $14(46.7 \%)$ & $6.89(3.24-14.65)$ & $<0.001^{* *}$ & $27(37.5 \%)$ & $5.39(3.14-9.26)$ & $<0.001^{* *}$ \\
\hline Surgeon & $36(4.9 \%)$ & $14(46.7 \%)$ & $27.01(11.73-62.15)$ & $<0.001^{* *}$ & $17(23.6 \%)$ & $10.41(5.12-21.18)$ & $<0.001^{* *}$ \\
\hline Physician & $21(2.9 \%)$ & $5(16.7 \%)$ & $8.56(2.91-25.23)$ & $<0.001^{* *}$ & $7(9.7 \%)$ & $4.96(1.93-12.73)$ & $0.001^{* *}$ \\
\hline Nurse & $20(2.7 \%)$ & $7(23.3 \%)$ & $16.11(5.88-44.15)$ & $<0.001^{* *}$ & $12(16.7 \%)$ & $16.28(6.40-41.37)$ & $<0.001^{* *}$ \\
\hline Orthotist & $4(0.5 \%)$ & $3(10.0 \%)$ & $77.78(7.83-772.35)$ & $<0.001^{* *}$ & $2(2.8 \%)$ & $9.39(1.30-67.67)$ & $0.026^{* *}$ \\
\hline Other & $9(1.2 \%)$ & 0 & 0 & NA & $1(1.4 \%)$ & $1.15(0.14-9.30)$ & 0.898 \\
\hline \multicolumn{8}{|l|}{ Foot disease history } \\
\hline Previous foot ulcer & $72(9.8 \%)$ & $21(70.0 \%)$ & $30.33(13.20-69.72)$ & $<0.001^{* *}$ & - & - & - \\
\hline \multicolumn{8}{|l|}{ Foot risk factors } \\
\hline Peripheral neuropathy & $160(22.0 \%)$ & $21(72.4 \%)$ & $10.65(4.62-24.56)$ & $<0.001^{* *}$ & $39(54.9 \%)$ & $5.39(3.24-8.95)$ & $<0.001^{* *}$ \\
\hline PAD & $153(21.0 \%)$ & $20(69.0 \%)$ & $9.44(4.20-21.20)$ & $<0.001^{* *}$ & $40(56.3 \%)$ & $6.20(3.72-10.34)$ & $<0.001^{* *}$ \\
\hline Foot deformity & $158(22.4 \%)$ & $17(65.4 \%)$ & $7.27(3.17-16.66)$ & $<0.001^{* *}$ & $29(42.0 \%)$ & $2.85(1.70-4.77)$ & $<0.001^{* *}$ \\
\hline
\end{tabular}

${ }^{*} p<0.2 ;{ }^{* *} p<0.05$; CI: confidence interval; GP: general practitioner; IQR: interquartile range; PAD: peripheral arterial disease; SD: standard deviation.

$51(7.2 \%(5.5-9.4 \%))$ with diabetes and $133(18.8 \%$ (16.2-21.9\%)) over 60 years old (Table 5). After univariate analysis, 18 explanatory variables were associated with foot deformity (all, $p<0.05$ ) (Table 5). No confounders were identified. Foot deformity was independently associated with older age groups (61-80 years $(4.7(1.8-12.2))$ and $81+$ years (5.7 (2.0-15.7))), PN (2.2 (1.4-2.4)), past foot treatment by a podiatrist (2.1 (1.4-3.1)), and mobility impairment (2.0 (1.33.1)) (all, $p<0.01$ ) (Table 8).

\section{Discussion}

This appears to be the first study to investigate a representative inpatient population for foot complications. Our findings indicate nearly half (46\%) of all inpatients had at least one foot complication that places them at risk of developing active foot disease, including nearly a quarter (24\%) at higher risk with multiple foot complications and a tenth (11\%) at very high risk of developing active foot disease with a history of previous foot disease. Inpatients with diabetes had significantly higher proportions of all foot complications than those without diabetes; however, interestingly, there were more patients with foot complications that did not have diabetes than did have diabetes due to the greater overall proportion of inpatients without diabetes. Foot complications in inpatients were associated with older age, males, indigenous peoples, diabetes, cerebrovascular accident (CVA) history, mobility impairment, other foot complications, and past foot treatment. Overall, these findings suggest that foot complications are relatively common in inpatient populations and also have common factors independently associated with them in both diabetes and nondiabetes inpatients.

To the best of our knowledge, the only foot complication to have been previously investigated in a representative inpatient population was PAD [3,4]. Our $21 \%$ prevalence for PAD seemed low compared to the $29 \%$ and $36 \%$ reported in the two previous similar studies [3, 20, 21]; however, an interrogation of these studies suggests closer alignment. The previous studies used an ankle-brachial index to diagnose PAD in inpatients over 40 years, whereas our study used toe systolic pressures to diagnose PAD in inpatients over 18 years $[20,21]$. Our equivalent PAD prevalence for our subgroup of inpatients over 40 years of age was 24\% (149/623), and toe pressures have been found to decrease false positive PAD identification compared with the ankle brachial indices $[13,15,16]$. These methodological differences 
TABLE 3: Independent factors associated with previous amputations (odds ratios [95\% CI]).

\begin{tabular}{|c|c|c|c|c|}
\hline Risk factor & Unadjusted & $p$ value & Adjusted $^{\mathrm{a}}$ & $p$ value \\
\hline CVA history & $4.83(1.48-15.74)$ & $0.009^{*}$ & $6.85[1.86-25.21]$ & $0.004^{*}$ \\
\hline Previous foot ulcer & $17.92(6.51-49.29)$ & $<0.001^{*}$ & $22.01[6.89-70.38]$ & $<0.001^{*}$ \\
\hline Foot deformity & $4.50(1.70-11.90)$ & $0.002^{*}$ & 5.59 [1.89-16.55] & $0.002^{*}$ \\
\hline Surgeon past foot treatment & $8.09(2.50-26.20)$ & $<0.001^{*}$ & $10.73[2.87-40.15]$ & $<0.001^{*}$ \\
\hline Model 1 results & $\begin{array}{c}\text { Pseudo } R^{2}: 0.447 \\
\text { Omnibus: } \mathrm{df}=4, p<0.001\end{array}$ & $\begin{array}{l}\text { Missing: } 29(4.0 \%) \\
\text { H\&L: } p=0.955\end{array}$ & $\begin{array}{c}\text { Pseudo } R^{2}: 0.516 \\
\text { Omnibus: } \mathrm{df}=8, p<0.001\end{array}$ & $\begin{array}{l}\text { Missing: } 50(6.8 \%) \text {; } \\
\text { H\&L: } p=0.628\end{array}$ \\
\hline
\end{tabular}

TABLE 4: Independent factors associated with previous foot ulcers (odds ratios [95\% CI]).

\begin{tabular}{lcccc}
\hline Risk factor & Unadjusted & $p$ value & Adjusted $^{\mathrm{a}}$ & $p$ value $^{2}$ \\
\hline Vision impairment & $2.10(1.09-4.03)$ & $0.026^{*}$ & $1.89(0.95-3.77)$ & 0.069 \\
PN & $3.17(1.81-5.56)$ & $<0.001^{*}$ & $3.75(2.06-6.85)$ & $<0.001^{*}$ \\
PAD & $3.77(2.15-6.62)$ & $<0.001^{*}$ & $3.88[2.14-7.06]$ & $<0.001^{*}$ \\
Podiatry past foot treatment & $3.16(1.80-5.55)$ & $<0.001^{*}$ & $2.88(1.59-5.22)$ & $<0.001^{*}$ \\
Nurse past foot treatment & $8.45(2.88-24.84)$ & $<0.001^{*}$ & $18.80(5.15-68.66)$ & $<0.001^{*}$ \\
\hline \multirow{2}{*}{ Model 1 results } & Pseudo $R^{2}: 0.305$ & Missing: $9(1.2 \%) ;$ & Pseudo $R^{2}: 0.364$ & Missing: $31(4.2 \%) ;$ \\
& Omnibus: $\mathrm{df}=5, p<0.001$ & H\&L: $p=0.154$ & Omnibus: df $=13, p<0.001$ & H\&L $p=0.601$ \\
\hline
\end{tabular}

${ }^{*} p<0.05$. ${ }^{a}$ Adjusted for identified confounders of geographical remoteness and socioeconomic status; pseudo $R^{2}$ : Nagelkerke $R^{2}$; omnibus: omnibus tests of model coefficients; df: degrees of freedom; missing: excluded cases with any missing data; H\&L: Hosmer and Lemeshow test; PAD: peripheral arterial disease; PN: peripheral neuropathy.

appear to explain the differences in our PAD prevalence findings compared to previous findings and suggest our findings are generalisable. We also found that diabetes patients (35\%) had much higher proportions of PAD than nondiabetes patients $(17 \%)$ which is also consistent with previous literature $[20,21]$.

Although previous amputation, previous foot ulceration, $\mathrm{PN}$, and foot deformity have not been previously investigated in representative inpatient populations, our findings are generally consistent to those reported for diabetes and geriatric inpatient populations $[3,4]$. Previous amputation prevalence reported by other studies were $1-8 \%$ within diabetes inpatients $[4,22,23]$ and $0-7 \%$ within over 60 years old $[4,7,24]$, which were similar to our findings of $9 \%$ and 6\% (24/433), respectively. Previous foot ulcer prevalence reported by other studies was $12-20 \%$ within diabetes inpatients $[4,25-27]$ and $1-15 \%$ within over 60 years old $[4,6,24]$ which again were similar to our findings of $20 \%$ and $11 \%$ (48/433), respectively. PN prevalence reported by other studies was $12-81 \%$ within diabetes inpatients $[4,9,28]$ and $26 \%$ within over 60 years old $[5,6]$, which were again similar to our findings of $43 \%$ and $29 \%$ (124/433), respectively. Although the $43-50 \%$ foot deformity prevalence has only been previously reported in geriatric inpatients $[4,7,29]$, and was much higher than our geriatric finding of $31 \%$ (133/433), this was likely explained by the different foot deformity definitions used between our study and the previous studies. Our study required at least three clinical characteristics of a foot deformity to be present to be defined as a foot deformity $[13,17]$, whereas previous studies required a much lower threshold of diagnosis with just one-foot deformity characteristic being required $[7,29]$. The overall interpretation of our diabetes and geriatric specific inpatient findings reassures us that our foot complication prevalence findings in representative inpatient populations are plausible and generalisable.

There has also been a general lack of literature investigating independent factors associated with foot complications in representative populations (inpatient or outpatients with and without diabetes). Yet interestingly, our representative inpatient findings for factors associated with foot complications were very similar to previously reported diabetes outpatient findings, even after we adjusted for diabetes. We found previous amputation was most strongly associated with previous foot ulcers which have been consistently identified in the diabetes literature to be the major precipitating risk factor for amputation $[12-14,16,17,30]$. Other factors identified in our study were foot deformity, CVA history, and past foot treatment by a surgeon. The association with foot deformity is most likely explained by a minor amputation procedure often producing a foot deformity in the remaining partial foot and a major amputation often precipitating a compensatory foot deformity in the remaining contralateral foot $[13,17]$. A CVA history has also been identified in other recent studies $[30,31]$ and may be explained by the similar macrovascular pathophysiology that occurs in both CVA and PAD which can subsequently result in amputation $[30,31]$. Lastly, past foot treatment by a surgeon in the previous year is perhaps not surprising considering amputation procedures can only be performed by surgeons; 


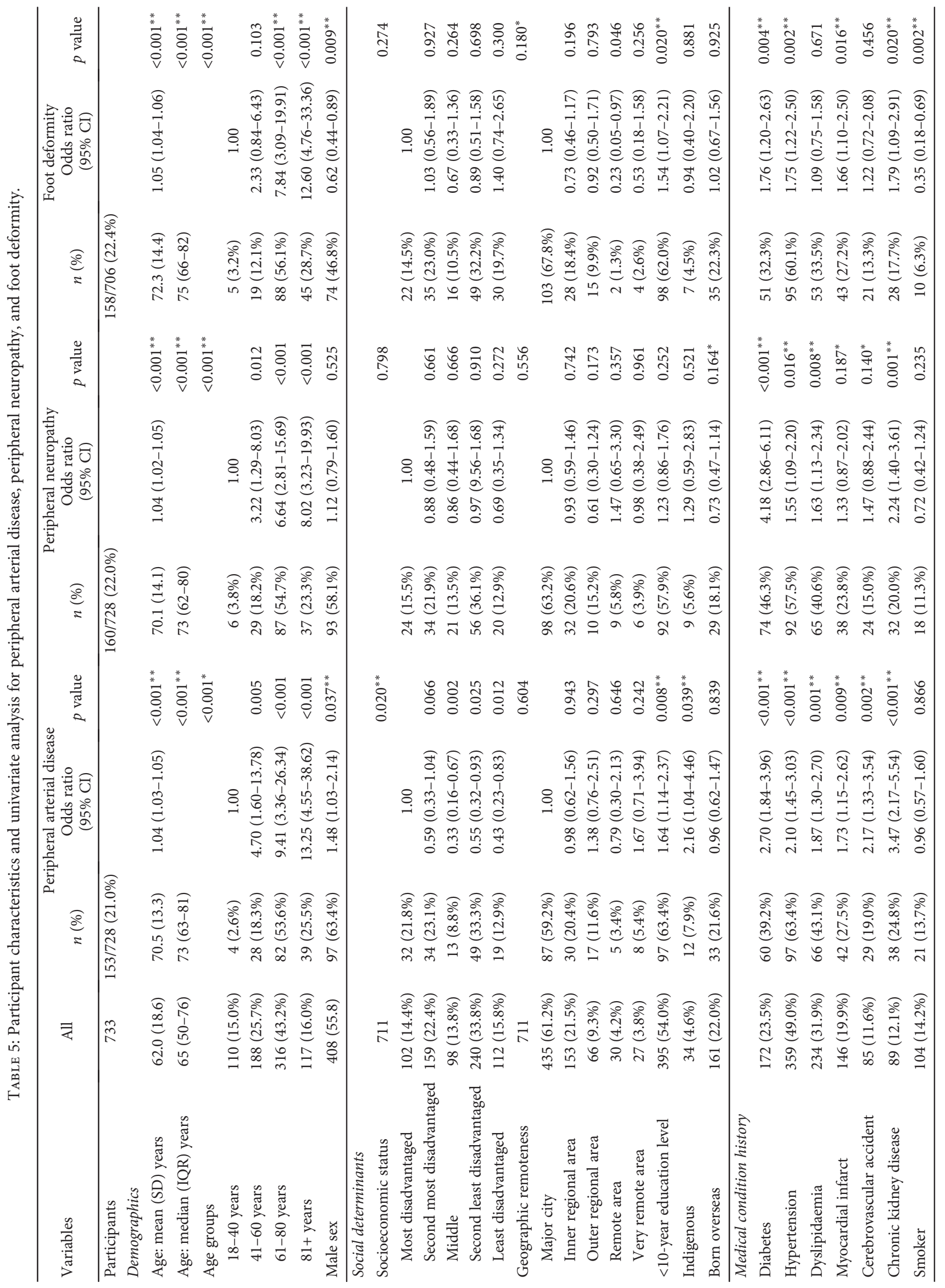




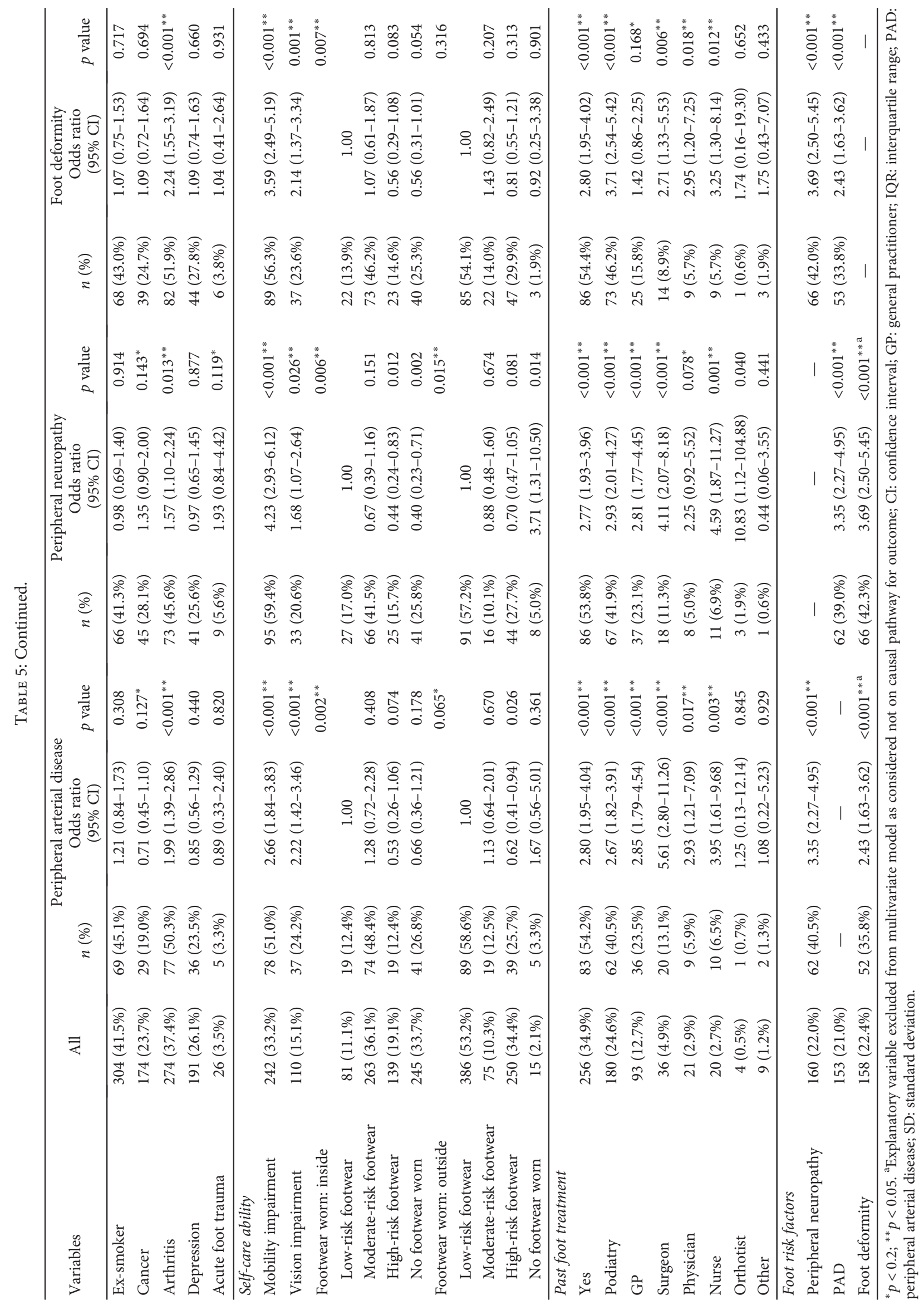


TABLE 6: Independent factors associated with peripheral arterial disease (odds ratios [95\% CI]).

\begin{tabular}{lcccc}
\hline Risk factor & Unadjusted & $p$ value & Adjusted $^{\text {a }}$ & \\
\hline Age groups & & $<0.001^{*}$ & Referent & \\
$\quad$ 18-40 years & Referent & & $<0.001^{*}$ \\
$\quad 41-60$ years & $4.98(1.64-15.14)$ & $0.005^{*}$ & $4.69(1.55-14.23)$ & $0.006^{*}$ \\
$\quad 61-80$ years & $10.42(3.56-30.51)$ & $<0.001^{*}$ & $8.94(3.04-26.33)$ & $<0.001^{*}$ \\
$\quad 81+$ years & $15.61(5.10-47.80)$ & $<0.001^{*}$ & $12.72(4.10-39.40)$ & $<0.001^{*}$ \\
Male sex & $1.55(1.04-2.32)$ & $0.031^{*}$ & $1.70(1.13-2.56)$ & $0.012^{*}$ \\
Indigenous & $3.23(1.40-7.43)$ & $0.006^{*}$ & $3.12(1.36-7.18)$ & $0.007^{*}$ \\
Cancer history & $0.52(0.32-0.85)$ & $0.009^{*}$ & $0.52(0.32-0.84)$ & $0.008^{*}$ \\
PN & $2.38(1.56-3.61)$ & $<0.001^{*}$ & $2.26(1.48-3.45)$ & $<0.001^{*}$ \\
Surgeon past foot treatment & $6.01(2.74-13.18)$ & $<0.001^{*}$ & Pseudo $R^{2}: 0.222$ & Missing: $11(1.5 \%)$; \\
\hline Model 1 results & Pseudo $R^{2}: 0.213$ & Missing: $11(1.5 \%) ;$ & Omnibus: $\mathrm{df}=9, p<0.001$ & H\&L: $p=0.038$ \\
\hline
\end{tabular}

${ }^{*} p<0.05 .{ }^{a}$ Adjusted for identified confounder of past podiatry treatment; pseudo $R^{2}$ : Nagelkerke $R^{2}$; omnibus: omnibus tests of model coefficients; df: degrees of freedom; missing: excluded cases with any missing data; H\&L: Hosmer and Lemeshow test; PN: peripheral neuropathy.

TABLE 7: Independent factors associated with peripheral neuropathy (odds ratios [95\% CI]).

\begin{tabular}{|c|c|c|c|c|}
\hline Risk factor & Unadjusted & $p$ value & Adjusted $^{\mathrm{a}}$ & $p$ value \\
\hline Age groups & & $0.007^{*}$ & & $0.008^{*}$ \\
\hline $18-40$ years & Referent & & Referent & \\
\hline $41-60$ years & $2.93(1.07-8.01)$ & $0.037^{*}$ & $2.77(1.01-7.62)$ & $0.048^{*}$ \\
\hline $61-80$ years & $4.66(1.80-12.08)$ & $0.002^{*}$ & $4.55(1.75-11.80)$ & $0.002^{*}$ \\
\hline $81+$ years & $4.73(1.70-13.15)$ & $0.003^{*}$ & $4.42(1.59-12.32)$ & $0.004^{*}$ \\
\hline Diabetes & $3.91(2.57-5.97)$ & $<0.001^{*}$ & $3.94(2.55-6.07)$ & $<0.001^{*}$ \\
\hline Mobility impairment & $3.37(2.22-5.11)$ & $<0.001^{*}$ & $3.41(2.24-5.20)$ & $<0.001^{*}$ \\
\hline PAD & $1.93(1.25-2.99)$ & $0.003^{*}$ & $2.08(1.33-3.25)$ & $0.001^{*}$ \\
\hline Outside footwear worn & & $0.017^{*}$ & & 0.055 \\
\hline Low risk & Referent & & Referent & \\
\hline Moderate risk & $0.58(0.29-1.15)$ & 0.117 & $0.57(0.28-1.13)$ & 0.108 \\
\hline High risk & $0.71(0.45-1.11)$ & 0.132 & $0.72(0.45-1.14)$ & 0.158 \\
\hline No footwear & $3.99(1.19-13.35)$ & $0.025^{*}$ & $3.01(0.85-10.65)$ & 0.088 \\
\hline Model 1 results & $\begin{array}{c}\text { Pseudo } R^{2}: 0.284 \\
\text { Omnibus: } \mathrm{df}=9, p<0.001\end{array}$ & $\begin{array}{l}\text { Missing: } 15(2.0 \%) \\
\text { H\&L: } p=0.100\end{array}$ & $\begin{array}{c}\text { Pseudo } R^{2}: 0.289 \\
\text { Omnibus: } \mathrm{df}=13, p<0.001\end{array}$ & $\begin{array}{l}\text { Missing: } 33(4.5 \%) \\
\text { H\&L: } p=0.189\end{array}$ \\
\hline
\end{tabular}

${ }^{*} p<0.05 .{ }^{a}$ Adjusted for identified confounder of geographical remoteness; pseudo $R^{2}$ : Nagelkerke $R^{2}$; omnibus: omnibus tests of model coefficients; df: degrees of freedom; missing: excluded cases with any missing data; H\&L: Hosmer and Lemeshow test; PAD: peripheral arterial disease.

however, we only captured past foot treatment for the year prior to hospitalisation and did not record the duration since the previous amputation was performed. Thus, further research would be required to determine how long people with a previous amputation maintain ongoing foot treatment with their surgeon after their procedure.

The independent factors we identified to be associated with previous foot ulceration were also consistent with those reported in the diabetes outpatient literature [12-14, 17, 32]. Our findings indicate that $\mathrm{PAD}, \mathrm{PN}$, and past foot treatment factors are important factors associated with foot ulceration, regardless of diabetes status $[13,17,32]$. Furthermore, a recent study also identified that $\mathrm{PAD}$ and $\mathrm{PN}$ were independently associated with active foot ulcers, regardless of diabetes; however, that study identified an association with past foot treatment by a surgeon rather than a podiatrist or nurse [2]. This adds weight to previous recommendations that best practice guidelines must emphasise the need for a podiatrist and nurse, in conjunction with a surgeon, to be part of the recommended outpatient multidisciplinary foot team to prevent foot ulcer inpatient admissions in diabetes and nondiabetes patients, rather than wait until the foot ulcer has healed to seek podiatry and nursing foot treatment as our findings suggest happens $[13,33,34]$. Nevertheless, these findings suggest, in both diabetes and nondiabetes populations, that these foot complications significantly increase 
TABLE 8: Independent factors associated with foot deformity (odds ratios [95\% CI]).

\begin{tabular}{|c|c|c|c|c|}
\hline Risk factor & Unadjusted & $p$ value & Adjusted $^{\mathrm{a}}$ & $\overline{p \text { value }}$ \\
\hline Age groups & & $<0.001^{*}$ & No confounders identified & \\
\hline $18-40$ years & Referent & & & \\
\hline $41-60$ years & $1.76(0.62-4.99)$ & 0.289 & & \\
\hline $61-80$ years & $4.67(1.79-12.17)$ & $0.002^{*}$ & & \\
\hline $81+$ years & $5.68(2.05-15.71)$ & $0.001^{*}$ & & \\
\hline Mobility impairment & $2.04(1.35-3.08)$ & $0.001^{*}$ & & \\
\hline $\mathrm{PN}$ & $2.20(1.44-2.36)$ & $<0.001^{*}$ & & \\
\hline Podiatry past foot treatment & $2.06(1.36-3.12)$ & $0.001^{*}$ & & \\
\hline Model 1 results & $\begin{array}{c}\text { Pseudo } R^{2}: 0.233 \\
\text { Omnibus: } \mathrm{df}=6, p<0.001\end{array}$ & $\begin{array}{l}\text { Missing: } 32(4.4 \%) \\
\text { H\&L: } p=0.938\end{array}$ & & \\
\hline
\end{tabular}
cases with any missing data; H\&L: Hosmer and Lemeshow test; PN: peripheral neuropathy.

the risk of developing future active foot disease (ulcers, infection, or ischaemia) which in turn increases the risk of potential hospitalisation and amputation.

PAD in our study was independently associated with older age, male gender, and $\mathrm{PN}$, which is consistent with previous outpatient literature [20, 21, 35]. Furthermore, our study identified Australian indigenous peoples as an independent factor associated with PAD which has also been identified by two previous diabetes-related Australian studies $[36,37]$. The independent associated factor of past surgical treatment for PAD is a welcome finding and suggests patients with $\mathrm{PAD}$ are being regularly assessed by vascular surgeons as recommended by best practice guidelines $[13,15,16]$, whereas our finding that a cancer history decreased the likelihood of having PAD is potentially a novel finding. It is perhaps most likely explained by cancer sufferers being hospitalised at younger ages [10], but more likely because our definition of cancer history was broad and included any cancers in the participant's history. Thus, it is recommended that any further research into this association captures data on different cancer types, severity, durations, and treatments to determine if the association is with particular types of cancers or treatments.

PN was found to be independently associated with older age, diabetes, and mobility impairment in our study, again all of which have been reported in the diabetes outpatient literature $[32,38]$. In contrast to best practice guideline recommendations that people with $\mathrm{PN}$ should receive regular foot monitoring by a podiatrist to prevent active foot disease, falls, or pressure injuries [13, 33], our study found no past foot treatment variables were independently associated with PN. PN has been reported to be the most important foot complication that precipitates the development of active foot disease, falls, and pressure injuries [3, 5-8]. Thus, our findings that one in every five inpatients, including nearly one in every two diabetes inpatients, has $\mathrm{PN}$ and is unlikely to have received any past foot treatment are concerning and highlight that further strategies are necessary to identify and monitor these patients in both the inpatient and outpatient settings $[13,33,34]$. The independent factors for foot deformity of older age, $\mathrm{PN}$, mobility impairment, and past podiatry treatment identified in our inpatient study are also consistent with similar outpatient literature [17, 38-40]. The association between foot deformity and PN has been consistently identified in the diabetes literature, and our findings suggest this link may be of similar importance in nondiabetes patients and should be investigated further in the future $[17,38-40]$.

Our overall findings suggest that foot complications that have been commonly reported to precipitate the development of active foot disease in the community are also present frequently in the inpatient population and have common factors independently associated with them, regardless of diabetes. Further research is recommended to more precisely determine the causal relationships for foot complications in nondiabetes populations in particular. It is recommended that policy makers and clinicians adopt simple hospital triage procedures that identify inpatients with these foot complications early to ensure that they do not develop into future active foot disease, falls, or pressure injuries whilst in hospital $[1,2,33,34]$. These procedures could be as simple as questioning all inpatients on admission, particularly those with diabetes or over 60 years of age, as to their previous foot disease history or using simple screening tools to identify PAD, PN, and foot deformity $[1,2,13,34]$. Nevertheless, clinicians and policy makers should continue to recommend inpatients identified with foot complications be assessed and managed whilst in an hospital and discharged to an outpatient multidisciplinary foot team for ongoing management in order to prevent potential hospitalisation from active foot disease, falls, and pressure injuries in the future $[1,2,33,34]$.

Additionally, although the independent associations between past foot treatment and most foot complications appear encouraging in our findings, this was not the case for PN. It could be argued that PN is the most critical foot complication that leads to active foot disease, falls, and pressure injuries, and thus, best practice guidelines need to better highlight that patients with $\mathrm{PN}$ require ongoing monitoring to ensure they can identify problems early to prevent possible future hospitalisation [13, 17, 32-34]. Lastly, it is recommended that policy makers incorporate 
these foot complications into their existing inpatient bedside audit programs alongside general diabetes, falls, and pressure injury bedside audit programs $[2,10,26]$. This should enable efficient monitoring of the influence of these foot complications on inpatient populations in the future and particularly their impact on inpatient adverse events $[1,2]$.

4.1. Strengths and Limitations. This study has a number of strengths and limitations which have been discussed elsewhere $[1,2]$. In brief, the strengths were this study investigated a highly representative Australian inpatient population $[1,2,10]$; data collectors were highly experienced, trained, and reliable in collecting validated and internationally agreed definitions of clinically diagnosed foot complications [11, 14]; and multivariate logistic regression models were used to adjust for confounding variables $[1,18,19]$. The limitations were this study was a cross-sectional study and was unable to test for causal relationships; excluded a large number of older cognitively impaired patients which may have led to a more conservative prevalence estimate of foot complications; had to aggregate minor and major previous amputations which are arguably the result of different causal pathways due to small numbers of both; did not exclude patients with active foot disease; and was a secondary analysis of a large dataset $[1,2]$ which increases the likelihood of type 1 statistical error $[18,19]$.

\section{Conclusions}

This study was the first to investigate multiple foot complications in a representative inpatient population. It identified that half of all inpatients had at least one-foot complication, with a quarter having multiple foot complications, which have been reported to be risk factors for the development active foot disease, pressure injuries, or falls whilst in hospital. The findings of this study suggest that regardless of having diabetes or not, common factors precipitate these foot complications. It is recommended that all inpatients are screened for these common foot complications on admission, particularly those with diabetes, and are managed accordingly to potentially prevent the large burden that foot disease already imposes on inpatient and outpatient populations.

\section{Conflicts of Interest}

The authors declare that there is no conflict of interest regarding the publication of this article.

\section{Acknowledgments}

This work was kindly supported by grant funding from Queensland Health (Queensland Government, Australia) and the Wound Management Innovation Cooperative Research Centre (Australia). The authors also wish to warmly acknowledge the tireless work of the Queensland's health-employed podiatrists and Queensland University of Technology podiatry students that undertook training, testing, and data collection for this project. Without their enthusiasm, this study would not have been possible.

\section{References}

[1] P. A. Lazzarini, S. E. Hurn, S. S. Kuys et al., "Direct inpatient burden caused by foot-related conditions: a multisite pointprevalence study," BMJ Open, vol. 6, article e010811, 2016.

[2] P. A. Lazzarini, S. E. Hurn, S. S. Kuys et al., "The silent overall burden of foot disease in a representative hospitalised population," International Wound Journal, vol. 14, no. 4, pp. 716728, 2017.

[3] P. A. Lazzarini, S. E. Hurn, M. Fernando et al., "Prevalence of foot disease and risk factors in general inpatient populations: a systematic review and meta-analysis," BMJ Open, vol. 5, article e008544, 2015.

[4] P. A. Lazzarini, S. E. Hurn, S. S. Kuys, M. C. Kamp, and L. Reed, "Foot-related conditions in hospitalised populations: a literature review," Wound Practice \& Research, vol. 24, no. 1, pp. 16-35, 2016.

[5] Z. Pataky, F. R. Herrmann, D. Regat, and H. Vuagnat, "The at-risk foot concerns not only patients with diabetes mellitus," Gerontology, vol. 54, no. 6, pp. 349-353, 2008.

[6] S. L. Burns, G. P. Leese, and M. E. McMurdo, "Older people and ill fitting shoes," Postgraduate Medical Journal, vol. 78, no. 920, pp. 344-346, 2002.

[7] R. L. Jessup, "Foot pathology and inappropriate footwear as risk factors for falls in a subacute aged-care hospital," Journal of the American Podiatric Medical Association, vol. 97, no. 3, pp. 213-217, 2007.

[8] B. J. Earl, P. A. Lazzarini, E. M. Kinnear, and P. L. Cornwell, "Prevalence of active foot disease and foot disease risk factors in a subacute inpatient rehabilitation facility: a crosssectional prevalence study," Journal of Foot and Ankle Research, vol. 7, no. 1, p. 41, 2014.

[9] M. He, J. Ma, D. Wang, J. Chang, and X. Yu, "The disease burden analysis of 295 inpatients with diabetes mellitus from Tongji hospital in China," Value in Health, vol. 13, no. 7, article A527, 2010.

[10] Australian Institute of Health \& Welfare (AIHW), Australian Hospital Statistics 2011-12, AIHW, Canberra, 2013, July 2016, http://www.aihw.gov.au/publication-detail/?id=60129543133.

[11] P. A. Lazzarini, V. Ng, E. M. Kinnear et al., “The Queensland high risk foot form (QHRFF) - is it a reliable and valid clinical research tool for foot disease?," Journal of Foot and Ankle Research, vol. 7, no. 1, p. 7, 2014.

[12] F. Crawford, C. Anandan, F. M. Chappell et al., "Protocol for a systematic review and individual patient data meta-analysis of prognostic factors of foot ulceration in people with diabetes: the international research collaboration for the prediction of diabetic foot ulcerations (PODUS)," BMC Medical Research Methodology, vol. 13, p. 22, 2013.

[13] National Health \& Medical Research Council (NHMRC) Guidelines, National Evidence-Based Guideline on Prevention, Identification and Management of Foot Complications in Diabetes (Part of the Guidelines on Management of Type 2 Diabetes), Baker IDI Heart \& Diabetes Institute, Melbourne, 2011, July 2016, https://www.nhmrc.gov.au/_files_ nhmrc/publications/attachments/diabetes_foot_full_guideline_ 23062011.pdf.

[14] W. J. Jeffcoate, S. A. Bus, F. L. Game, R. J. Hinchliffe, P. E. Price, and N. C. Schaper, "Reporting standards of studies and papers on the prevention and management of foot ulcers in diabetes: required details and markers of good quality," The 
Lancet Diabetes and Endocrinology, vol. 4, no. 9, pp. 781-788, 2016.

[15] J. L. Mills Sr., M. S. Conte, D. G. Armstrong et al., “The society for vascular surgery lower extremity threatened limb classification system: risk stratification based on wound, ischemia, and foot infection (WIfI)," Journal of Vascular Surgery, vol. 59, no. 1, pp. 220.e1-234.e2, 2014.

[16] N. C. Schaper, "Diabetic foot ulcer classification system for research purposes: a progress report on criteria for including patients in research studies," Diabetes/Metabolism Research and Reviews, vol. 20, Supplement 1, pp. S90-SS5, 2004.

[17] C. A. Abbott, A. L. Carrington, H. Ashe et al., "The NorthWest Diabetes Foot Care Study: incidence of, and risk factors for, new diabetic foot ulceration in a community-based patient cohort," Diabetic Medicine, vol. 19, pp. 377-384, 2002.

[18] B. G. Tabachnick and L. S. Fidell, Using Multivariate Statistics, Allyn and Bacon, Boston, MA, USA, Fifth edition, 2007.

[19] D. Hosmer and S. Lemeshow, Applied Logistic Regression, John Wiley \& Sons, New York, NY, USA, Second edition, 2000.

[20] S. Antonopoulos, S. Kokkoris, F. Stasini et al., "High prevalence of subclinical peripheral artery disease in Greek hospitalized patients," European Journal of Internal Medicine, vol. 16, no. 3, pp. 187-191, 2005.

[21] P. Lacroix, V. Aboyans, D. Voronin, A. Le Guyader, M. Cautrès, and M. Laskar, "High prevalence of undiagnosed patients with peripheral arterial disease in patients hospitalised for non-vascular disorders," International Journal of Clinical Practice, vol. 62, no. 1, pp. 59-64, 2008.

[22] C. Buckley, A. O'Farrell, C. Bradley et al., "Trends in hospital admissions for diabetes and diabetes-related lower extremity amputations in the Republic of Ireland over a five year period," Diabetologia, vol. 54, pp. S128-S1S9, 2011.

[23] D. Sano, H. Tieno, Y. Drabo, and A. Sanou, "Management of the diabetic foot, apropos of 42 cases at the Ougadougou University Hospital Center," Dakar Médical, vol. 43, no. 1, pp. 109-113, 1998.

[24] J. F. Reed III, "An audit of lower extremity complications in octogenarian patients with diabetes mellitus," The International Journal of Lower Extremity Wounds, vol. 3, no. 3, pp. 161-164, 2004.

[25] S. M. Lawrence, P. R. Wraight, D. A. Campbell, and P. G. Colman, "Assessment and management of inpatients with acute diabetes-related foot complications: room for improvement," Internal Medicine Journal, vol. 34, no. 5, pp. 229-233, 2004.

[26] G. Rayman, C. G. Taylor, R. Malik, and R. Hilson, "The national inpatient diabetes audit reveals poor levels of inpatient foot care," Diabetologia, vol. 53, article S406, 2010.

[27] G. Shabbier, S. Amin, I. Khattak, and R. Sadeeq ur, "Diabetic foot risk classification in a tertiary care teaching hospital of Peshawar," Journal of Postgraduate Medical Institute, vol. 24, no. 1, pp. 22-26, 2010.

[28] A. Adem, T. Demis, and Y. Feleke, "Trend of diabetic admissions in Tikur Anbessa and St. Paul's University Teaching Hospitals from January 2005-December 2009, Addis Ababa, Ethiopia," Ethiopian Medical Journal, vol. 49, no. 3, pp. 231238, 2011.

[29] L. K. Hung, Y. F. Ho, and P. C. Leung, "Survey of foot deformities among 166 geriatric inpatients," Foot \& Ankle, vol. 5, no. 4, pp. 156-164, 1985.
[30] M. Baba, W. A. Davis, P. E. Norman, and T. M. Davis, "Temporal changes in the prevalence and associates of diabetesrelated lower extremity amputations in patients with type 2 diabetes: the Fremantle Diabetes Study," Cardiovascular Diabetology, vol. 14, p. 152, 2015.

[31] J. J. van Netten, L. V. Fortington, R. J. Hinchliffe, and J. M. Hijmans, "Early post-operative mortality after major lower limb amputation: a systematic review of population and regional based studies," European Journal of Vascular and Endovascular Surgery, vol. 51, no. 2, pp. 248-257, 2016.

[32] R. J. Tapp, J. E. Shaw, M. P. D. Courten, D. W. Dunstan, T. A. Welborn, and P. Z. Zimmet, "Foot complications in type 2 diabetes: an Australian population-based study," Diabetic Medicine, vol. 20, no. 2, pp. 105-113, 2003.

[33] Scottish Intercollegiate Guidelines Network (SIGN), Management of Diabetes: A National Clinical Guideline, SIGN, Edinburgh, 2010, July 2016, http://www.sign.ac.uk/guidelines/ fulltext/116/.

[34] D. K. Wukich, D. G. Armstrong, C. E. Attinger et al., "Inpatient management of diabetic foot disorders: a clinical guide," Diabetes Care, vol. 36, no. 9, pp. 2862-2871, 2013.

[35] F. G. R. Fowkes, D. Rudan, I. Rudan et al., "Comparison of global estimates of prevalence and risk factors for peripheral artery disease in 2000 and 2010: a systematic review and analysis," Lancet, vol. 382, no. 9901, pp. 1329-1340, 2013.

[36] L. Maple-Brown, J. Cunningham, K. Dunne et al., "Complications of diabetes in urban indigenous Australians: the DRUID study," Diabetes Research and Clinical Practice, vol. 80, no. 3, pp. 455-462, 2008.

[37] Australian Institute of Health \& Welfare (AIHW), Diabetes: Australian Facts, AIHW, Canberra, 2008, July 2016, http:// www.aihw.gov.au/publication-detail/?id=6442468075.

[38] S. G. Bruce and T. K. Young, "Prevalence and risk factors for neuropathy in a Canadian first nation community," Diabetes Care, vol. 31, no. 9, pp. 1837-1841, 2008.

[39] H. B. Menz, T. K. Gill, A. W. Taylor, and C. L. Hill, "Predictors of podiatry utilisation in Australia: the North West Adelaide Health Study," Journal of Foot and Ankle Research, vol. 1, no. 1, p. 8, 2008.

[40] S. Nix, M. Smith, and B. Vicenzino, "Prevalence of hallux valgus in the general population: a systematic review and metaanalysis," Journal of Foot and Ankle Research, vol. 3, p. 21, 2010. 


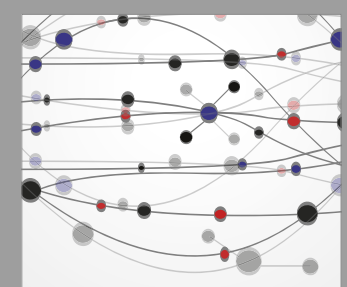

The Scientific World Journal
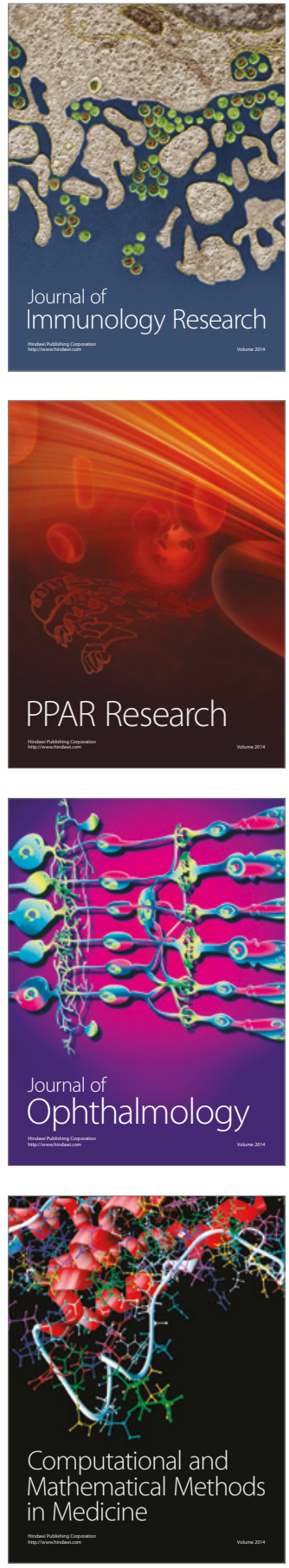



Gastroenterology Research and Practice
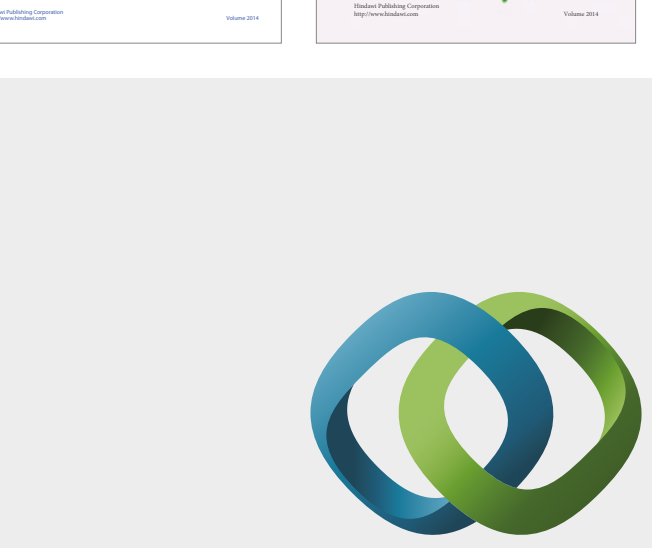

\section{Hindawi}

Submit your manuscripts at

https://www.hindawi.com
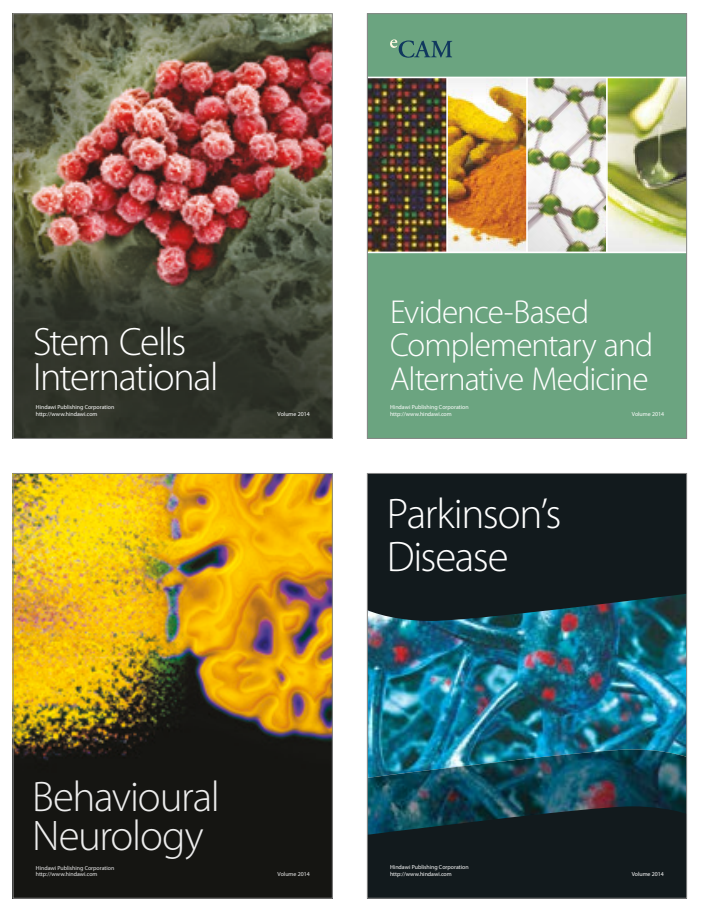
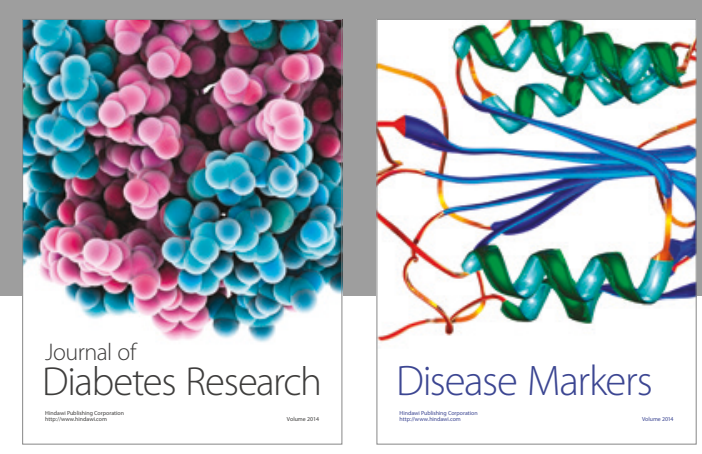

Disease Markers
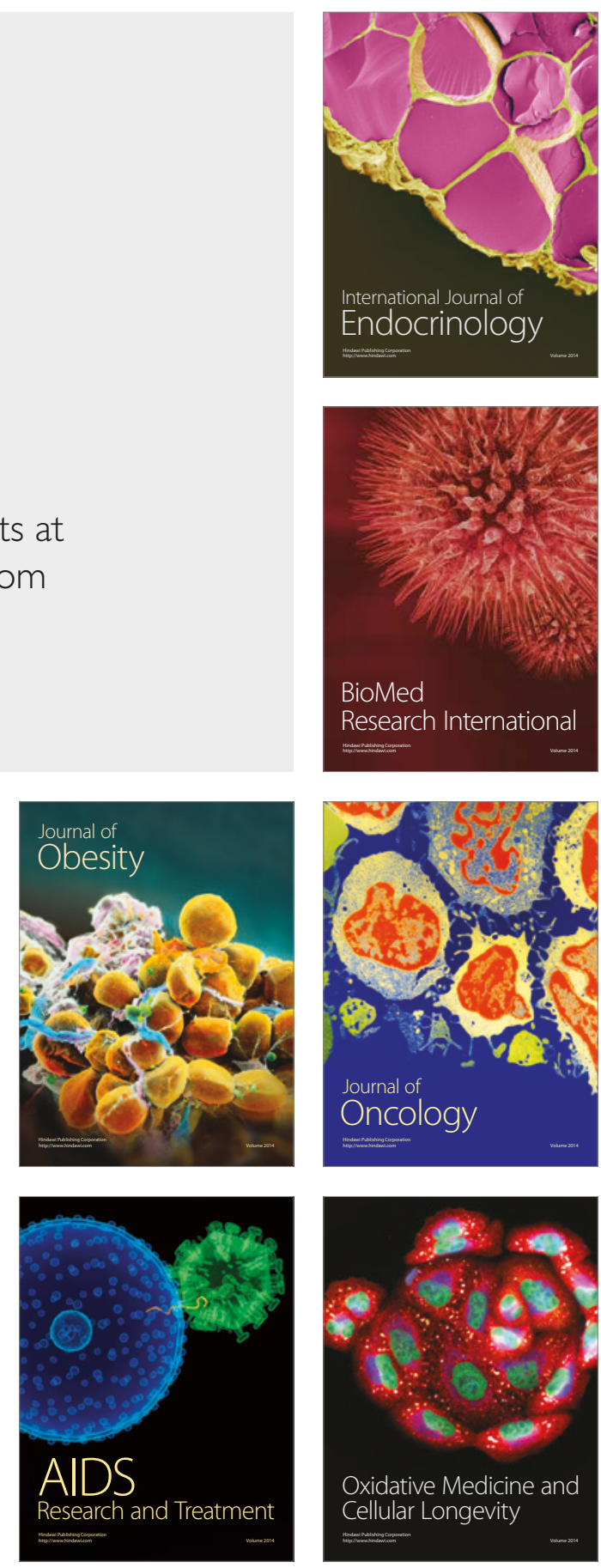